\title{
Nonresonance between the first two Eigencurves of Laplacian for a Nonautonomous Neumann Problem
}

Ahmed Sanhaj: Ahmed Dakkak

Sidi Mohamed Ben Abdellah University, Mathematics Physics and Computer Science, LSI, FP, Taza, Morocco

\begin{tabular}{l} 
A R T I C L E I N F O \\
\hline Article history: \\
Received: 25 May, 2017 \\
Accepted: 13 July, 2017 \\
Online: 29 December, 2017 \\
\hline Keywords: \\
Laplacian \\
Nonresonance \\
Neumann problem \\
\hline
\end{tabular}

\section{Introduction}

Let $\Omega$ be a bounded domain of $\mathbb{R}^{N}(N \geq 1)$, with $C^{1,1}$ boundary and let $v$ be the outward unit normal vector on $\partial \Omega$.

D. Del Santo and P. Omari, have studied in [1] the Dirichlet problem

$$
\begin{cases}-\Delta u=g(u)+h(x) & \text { in } \Omega \\ u=0 & \text { on } \partial \Omega,\end{cases}
$$

They have proved the existence of nontrivial weak solutions for this problem for every given $h \in L^{p}(\Omega)$ under some assumptions on the function $g$. In the case of Neumann elliptic problem J.-P. Gossez and P. Omari, have considered in [2] the following problem

$$
\begin{cases}-\Delta u=g(u)+h(x) & \text { in } \Omega \\ \frac{\partial u}{\partial v}=0 & \text { on } \partial \Omega,\end{cases}
$$

They have shown the existence of weak solutions for this problem for every given $h \in L^{\infty}(\Omega)$ under some conditions on function $g$. A.Dakkak and A. Anane studied in [3] the existence of weak solutions for the problem

$$
\left\{\begin{array}{rlrl}
-\Delta u & =\lambda_{2} m(x) u+g(u)+h(x) & & \text { in } \Omega \\
\frac{\partial u}{\partial v}=0 & \text { on } \partial \Omega,
\end{array}\right.
$$

where $\lambda_{2}=\lambda_{2}(m)$ is the second eigenvalue of $-\Delta$ with weight $m$, with $m \in M^{+}(\Omega)=$ $\left\{m \in L^{\infty}(\Omega): \operatorname{meas}(\{x \in \Omega: m(x)>0\}) \neq 0\right\}$.

We investigate in the present work the following Neumann elliptic problem

$(\mathcal{P}) \begin{cases}-\Delta u=\alpha m_{1}(x) u+m_{2}(x) g(u)+h(x) & \text { in } \Omega, \\ \frac{\partial u}{\partial v}=0 & \text { on } \partial \Omega .\end{cases}$

where $-\Delta$ is the Laplacian operator. The functions $m_{1}, m_{2} \in M^{+}(\Omega), h \in L^{\infty}(\Omega), g: \mathbb{R} \rightarrow \mathbb{R}$ is a continuous function and $\alpha$ is a real parameter such that $\alpha \geq \lambda_{2}\left(m_{1}\right)$ or $\alpha \leq \lambda_{-} 2\left(m_{1}\right)$, with $\lambda_{-} 2\left(m_{1}\right)=-\lambda_{2}\left(-m_{1}\right)$. By a solution of $(\mathcal{P})$ we mean a function $u \in H^{1}(\Omega) \cap$ $L^{\infty}(\Omega)$, such that

$$
\int_{\Omega} \nabla u \nabla w=\int_{\Omega}\left(\alpha m_{1} u+m_{2} g(u)+h\right) w
$$

for every $w \in H^{1}(\Omega)$.

This paper is organized as follows. In section 2, we recall some results that we will use later. Section 3 is concerned with the existence of principal eigencurve of the Laplacian operator with Neumann boundary conditions. In section 4, we show a theorem of nonresonance between the first and second eigenvalue (see theorem 2). In section 5, we prove the nonresonance between the first two eigencurves for problem $(\mathcal{P})$.

\footnotetext{
${ }^{*}$ Corresponding Author: Ahmed Sanhaji, Laboratory-LSI- sidi Mohamed ben Abdellah University, polydisciplinary, Faculty of TAZA, MOROCCO, \& ahmed.sanhaji1@usmba.ac.ma
} 


\section{Preliminary}

Let us briefly recall some properties of the spectrum of $-\Delta$ with weight and with Neumann boundary condition to be used later. Let be $\Omega$ a smooth bounded domain in $\mathbb{R}^{N}(N \geq 1)$ and let $m \in M^{+}(\Omega)$. the eigenvalue problem is

$$
\begin{cases}-\Delta u=\lambda m(x) u & \text { in } \quad \\ \frac{\partial u}{\partial v}=0 & \text { on } \quad \partial \Omega,\end{cases}
$$

this spectrum contains a sequence of nonnegative eigenvalues $\left(\lambda_{n}\right)_{n>0}$ given by

$$
\frac{1}{\lambda_{n}}=\frac{1}{\lambda_{n}(m)}=\sup _{K \in \Gamma_{n}} \min _{u \in K} \frac{\int_{\Omega} m u^{2}}{\int_{\Omega}|\nabla u|^{2}},
$$

where $\Gamma_{n}=\{K \subset S: \mathrm{K}$ is symetric, compact and $\gamma(K) \geq n\}$ $S$ is the unit sphere of $H^{1}(\Omega)$ and $\gamma$ is the genus function. This formulation can be found in [4], the sequence $\left(\lambda_{n}\right)_{n>0}$ verify:

i) $\lambda_{n} \rightarrow+\infty$ as $n \rightarrow+\infty$

ii) If $m$ change its sign in $\Omega$ and $\int_{\Omega} m d x<0$, then the first eigenvalue defined by

$$
\lambda_{1}(m)=\inf \left\{\int_{\Omega}|\nabla u|^{2}, u \in H^{1}(\Omega) / \int_{\Omega} m u^{2} d x=1\right\}
$$

it is known that $\lambda_{1}(m)$ is $>0$, simple and the associated eigenfunction $\varphi_{1}$ can be chosen such that $\varphi_{1}>0$ in $\Omega$ and $\left\|\varphi_{1}\right\|_{H^{1}}=1$ hold. Moreover $\lambda_{1}(m)$ is isolated in the spectrum, which allows to define the second positive eigenvalue $\lambda_{2}(m)$ as

$\lambda_{2}(m)=\min \left\{\lambda \in \mathbb{R}: \lambda\right.$ is eigenvalue and $\left.\lambda>\lambda_{1}(m)\right\}$

it is also known that any eigenfunction associated to a positive eigenvalue different from $\lambda_{1}(m)$ changes sign in $\Omega$.

iii) $\lambda_{1}(m)$ is strictly monotone decreasing with respect to $m$ (i.e. $m \supsetneqq m^{\prime}$ implies $\lambda_{1}(m)>\lambda_{1}\left(m^{\prime}\right)$ ).

Throughout this work, the functions $m_{1}$ and $m_{2}$ satisfies the following assumptions:

(A) $\quad m_{1}, m_{2} \in M^{+}(\Omega)$ and $e s s \inf _{\Omega} m_{2}>0$.

Proposition 1 ([3]). Let $m, m^{\prime} \in M^{+}(\Omega)$.

1. If $m \leq m^{\prime}$, then $\lambda_{2}(m) \geq \lambda_{2}\left(m^{\prime}\right)$.

2. $\lambda_{2}: m \rightarrow \lambda_{2}(m)$ is continuous in $\left(M^{+}(\Omega),\|.\|_{\infty}\right)$.

Proposition 2 ([3]). Let $\left(m_{k}\right)_{k}$ be a sequence in $M^{+}(\Omega)$ such that $m_{k} \rightarrow m$ in $L^{\infty}(\Omega)$. then $\lim _{k \rightarrow \infty} \lambda_{2}\left(m_{k}\right)=+\infty$ if and only if $m \leq 0$ almost everywhere in $\Omega$.

\section{Existence of the second eigen- curve of the $-\Delta$ with weighs in the Neumann case}

The second eigencurve of the $-\Delta$ with weighs is defined as a set $\mathcal{C}_{2}$ of those $(\alpha, \beta) \in \mathbb{R}^{2}$ such that the fol- lowing Neumann problem

$$
\begin{cases}-\Delta u=\alpha m_{1}(x) u+\beta m_{2}(x) u & \text { in } \quad \Omega \\ \frac{\partial u}{\partial v}=0 & \text { on } \quad \partial \Omega,\end{cases}
$$

has a nontrivial solution $u \in H^{1}(\Omega)$ (i.e. $\mathcal{C}_{2}=$ $\left.\left\{(\alpha, \beta) \in \mathbb{R}^{2} ; \lambda_{2}\left(\alpha m_{1}+\beta m_{2}\right)=1\right\}\right)$, where $m_{1}$ and $m_{2}$ satisfies the condition (A). For more details see $[5,6]$. The purpose in this section is to study the following problem: For $\beta<0$, we prove the existence and the uniqueness of reel $\alpha_{2}^{+}(\beta)$ such that $\left.\left(\alpha_{2}^{+}(\beta), \beta\right) \in \mathcal{C}_{2}\right)$. Given $m \in M^{+}(\Omega)$, we denote by $\Omega_{m}^{+}=$ $\{x \in \Omega ; m(x)>0\}$ and $\Omega_{m}^{-}=\{x \in \Omega ; m(x)<0\}$.

Remark 1 Let $(\alpha, \beta) \in \mathcal{C}_{2}$.

1. If $\alpha>\lambda_{2}\left(m_{1}\right)$, then we have $\beta<0$.

2. If meas $\left(\Omega_{m}^{-}\right)>0$ and $\alpha<\lambda_{-} 2\left(m_{1}\right)$, we have $\beta<0$.

Indeed, assume by contradiction if $\alpha>\lambda_{2}\left(m_{1}\right)$ and $\beta \geq 0$, then

$$
\alpha m_{1} \leq \alpha m_{1}+\beta m_{2},
$$

using the monotony property of $\lambda_{2}$, we obtain

$$
\lambda_{2}\left(\alpha m_{1}+\beta m_{2}\right) \leq \lambda_{2}\left(\alpha m_{1}\right)=\frac{\lambda_{2}\left(m_{1}\right)}{\alpha}<1,
$$

since, $(\alpha, \beta) \in \mathcal{C}_{2}$, we have $\lambda_{2}\left(\alpha m_{1}+\beta m_{2}\right)=1$, thus necessarily $\beta<0$. The proof of the second assertion is similar.

Theorem 1 Let $m_{1}, m_{2}$ satisfy $(\boldsymbol{A})$, then we have:

i) For all $\beta<0$, there exists $\alpha_{2}^{+}(\beta)>\lambda_{2}\left(m_{1}\right)$ such that $\left(\alpha_{2}^{+}(\beta), \beta\right) \in \mathcal{C}_{2}$.

ii) If meas $\left(\Omega_{m_{1}}^{-}\right)>0$, then for all $\beta<0$, there exists $\alpha_{2}^{-}(\beta)<\lambda_{-} 2\left(m_{1}\right)$ such that $\left(\alpha_{2}^{-}(\beta), \beta\right) \in \mathcal{C}_{2}$.

Proof To prove $\mathbf{i}$ ), we consider $\beta<0$ and we define $\alpha_{2}^{+}(\beta)$ as follows

$$
\frac{1}{\alpha_{2}^{+}(\beta)}=\sup _{K \in \Gamma_{2}} \inf _{u \in K} \frac{\int_{\Omega} m_{1} u^{2}}{\int_{\Omega}|\nabla u|^{2}-\beta \int_{\Omega} m_{1} u^{2}}
$$

by definition of $\alpha_{2}^{+}(\beta)$ and, using the fact that for any eigenfunction associated to $\lambda_{2}\left(m_{1}\right)$ changes sign in $\Omega$, we obtain that there exists eigenfunction $u$ which change sign in $\Omega$ such that

$$
\int_{\Omega} \nabla u \cdot \nabla v-\beta \int_{\Omega} m_{2} u v=\int_{\Omega} \alpha_{2}^{+}(\beta) m_{1} v,
$$

for all $v \in H^{1}(\Omega)$, we deduce also that, if $w \in H^{1}(\Omega)$ is eigenfunction of operator $-\Delta()-.\beta m_{2}($.$) which change$ singe in $\Omega$ with the corresponding eigenvalue $\lambda>0$, then $\lambda \geq \alpha_{2}^{+}(\beta)$. In view of the $\sqrt{6}$, we have

$$
\int_{\Omega} \nabla u \cdot \nabla v=\int_{\Omega}\left(\alpha_{2}^{+}(\beta) m_{1}+\beta m_{2} u\right) v \quad \text { for all } v \in H^{1}(\Omega)
$$


it follows that the real 1 is eigenvalue of $-\Delta$ with weight $\left(\alpha_{2}^{+}(\beta)+\beta m_{2}\right)$, since the corresponding eigenfunction u change singe in $\Omega$, we conclude that

$$
\lambda_{2}\left(\alpha_{2}^{+}(\beta) m_{1}+\beta m_{2}\right) \leq 1
$$

On the other hand, using (5) for all $K \in \Gamma_{2}$, there exists $u_{K} \in K$ such that

$$
\begin{aligned}
\min _{u \in K} & \frac{\int_{\Omega} m_{1} u^{2}}{\int_{\Omega}|\nabla u|^{2}-\beta \int_{\Omega} m_{1} u^{2}} \\
& =\frac{\int_{\Omega} m_{1} u_{K}^{2}}{\int_{\Omega}\left|\nabla u_{K}\right|^{2}-\beta \int_{\Omega} m_{2} u_{K}^{2}} \leq \frac{1}{\alpha_{2}^{+}(\beta)},
\end{aligned}
$$

it follows that

$$
\frac{\int_{\Omega}\left(\alpha_{2}^{+}(\beta) m_{1}+\beta m_{2}\right) u_{K}^{2}}{\int_{\Omega}\left|\nabla u_{K}\right|^{2}} \leq 1 .
$$

So that

$$
\begin{aligned}
\min _{u \in K} & \frac{\int_{\Omega}\left(\alpha_{2}^{+}(\beta) m_{1}+\beta m_{2}\right) u^{2}}{\int_{\Omega}|\nabla u|^{2}} \\
& \leq \frac{\int_{\Omega}\left(\alpha_{2}^{+}(\beta) m_{1}+\beta m_{2}\right) u_{K}^{2}}{\int_{\Omega}\left|\nabla u_{K}\right|^{2}} \leq 1 \text { for all } K \in \Gamma_{2},
\end{aligned}
$$

this implies

$$
\sup _{K \in \Gamma_{2}} \min _{u \in K} \frac{\int_{\Omega}\left(\alpha_{2}^{+}(\beta) m_{1}+\beta m_{2}\right) u^{2}}{\int_{\Omega}|\nabla u|^{2}} \leq 1 .
$$

Since

$\frac{1}{\lambda_{2}\left(\alpha_{2}^{+}(\beta) m_{1}+\beta m_{2}\right)}=\sup _{K \in \Gamma_{2}} \min _{u \in K} \frac{\int_{\Omega}\left(\alpha_{2}^{+}(\beta) m_{1}+\beta m_{2}\right) u^{2}}{\int_{\Omega}|\nabla u|^{2}}$

we deduce that

$$
\lambda_{2}\left(\alpha_{2}^{+}(\beta) m_{1}+\beta m_{2}\right) \geq 1
$$

By combining (7) and (8), we obtain

$$
\lambda_{2}\left(\alpha_{2}^{+}(\beta) m_{1}+\beta m_{2}\right)=1
$$

Let $\gamma>0$ such that $\lambda_{2}\left(\gamma m_{1}+\beta m_{2}\right)=1$, there exists eigenfunction $\omega$ change singe in $\Omega$ and

$$
\int_{\Omega} \nabla u \cdot \nabla \omega=\int_{\Omega}\left(\gamma m_{1}+\beta m_{2} u\right) \omega \quad \forall \omega \in H^{1}(\Omega)
$$

hence

$$
\int_{\Omega} \nabla u \cdot \nabla \omega-\int_{\Omega} \beta m_{2} u \omega=\gamma \int_{\Omega} m_{1} \omega \quad \forall \omega \in H^{1}(\Omega)
$$

from (9), we obtain that $\gamma$ is eigenvalue of the operator $-\Delta()-.\beta m_{2}($.$) with weight m_{1}$, since the eigenfunction $\omega$ change singe, we conclude that

$$
\gamma \geq \alpha_{2}^{+}(\beta) \text {. }
$$

Assume by contradiction that $\gamma>\alpha_{2}^{+}(\beta)$, then

$$
\frac{1}{\gamma}<\frac{1}{\alpha_{2}^{+}(\beta)}=\sup _{K \in \Gamma_{2}} \min _{u \in K} \frac{\int_{\Omega} m_{1} u^{2}}{\int_{\Omega}|\nabla u|^{2}-\beta \int_{\Omega} m_{2} u^{2}},
$$

by the inequality above we deduce that there exists $K_{0} \in \Gamma_{2}$ such that

$$
\frac{1}{\gamma}<\min _{u \in K_{0}} \frac{\int_{\Omega} m_{1} u^{2}}{\int_{\Omega}|\nabla u|^{2}-\beta \int_{\Omega} m_{2} u^{2}},
$$

since $K_{0}$ is compact, we conclude that, there exists $u_{0} \in K_{0}$

$$
\frac{1}{\gamma}<\frac{\int_{\Omega} m_{1} u_{0}^{2}}{\int_{\Omega}\left|\nabla u_{0}\right|^{2}-\beta \int_{\Omega} m_{2} u_{0}^{2}},
$$

hence

$$
1<\min _{u \in K_{0}} \frac{\int_{\Omega}\left(\gamma m_{1}+\beta m_{2}\right) u_{0}^{2}}{\int_{\Omega}\left|\nabla u_{0}\right|^{2}}
$$

it follows that

$$
1<\sup _{K \in \Gamma_{2}} \min _{u \in K_{0}} \frac{\int_{\Omega}\left(\gamma m_{1}+\beta m_{2}\right) u_{0}^{2}}{\int_{\Omega}\left|\nabla u_{0}\right|^{2}}=\frac{1}{\lambda_{2}\left(\gamma m_{1}+\beta m_{2}\right)}=1,
$$

which gives a contradiction, thus we have $\gamma=\alpha_{2}^{+}(\beta)$.

\section{Nonresonance between the first and second eigenvalue}

In this section we are interesting to the study of the existence results for the following Neumann problem

$\left(\mathcal{P}_{2}\right) \begin{cases}-\Delta u=\lambda_{2} m_{1}(x) u+m_{2}(x) g(u)+h(x) & \text { in } \Omega \\ \frac{\partial u}{\partial v}=0 & \text { on } \partial \Omega,\end{cases}$

where $\lambda_{2}=\lambda_{2}\left(m_{1}\right)$ is the second eigenvalue of $-\Delta$ with weight $m_{1}$ under the Neumann boundary condition.

Lemma 1 Let $m_{1}, m_{2} \in M^{+}(\Omega)$. Assume that $(\boldsymbol{A})$ is verified, then there exists a unique real $c>0$ such that

$$
\lambda_{1}\left(\lambda_{2} m_{1}-c m_{2}\right)=1
$$

Proof Put $a=\frac{e s s \inf _{\Omega} \lambda_{2} m_{1}}{e s \operatorname{sinf}_{\Omega} m_{2}}$ and $b=\frac{e s s \sup _{\Omega} \lambda_{2} m_{1}}{e s s \inf _{\Omega} m_{2}}$ since $m$ is a nonconstant function, then we have $a<b$. So for $t \in\left[a, b\left[\right.\right.$ we consider the weight $m_{t}=\lambda_{2} m_{1}-$ $t m_{2}$ and the corresponding increasing and continuous function:

$$
\begin{aligned}
f:[a, b[ & \rightarrow \mathbb{R} \\
t & \mapsto \lambda_{1}\left(\lambda_{2} m_{1}-t m_{2}\right)
\end{aligned}
$$

which satisfy $f(a)=0$ and $\lim _{t \rightarrow b} f(t)=+\infty$. According to be strict monotony property of $\lambda_{1}$ with weights we observe that $f$ is strictly increasing on $\left[a^{\prime}, b[\right.$ where $a^{\prime}=\max \{t: f(t)=0\}$.

Therefore, $f\left(a^{\prime}\right)=0 \leq f(0)=\lambda_{1}\left(\lambda_{2} m_{1}\right)<\lambda_{2}\left(\lambda_{2} m_{1}\right)=$ 1 , so the conclusion follows from the intermediate values theorem. 
Theorem 2 Let $m_{1}, m_{2} \in M^{+}(\Omega)$. Assume that the weights $m_{1}$ and $m_{2}$ satisfy $(\boldsymbol{A})$ and the function $g$ satisfy the following hypotheses

$$
\begin{array}{r}
-c \leq \liminf _{s \rightarrow \pm \infty} \frac{g(s)}{s} \leq \limsup _{s \rightarrow \pm \infty} \frac{g(s)}{s} \leq 0 \\
-c<\limsup _{|s| \rightarrow \infty} \frac{2 G(s)}{s^{2}} ; \liminf _{s \rightarrow+\infty o r-\infty} \frac{2 G(s)}{s^{2}}<0
\end{array}
$$

where $c$ is given in 10 , then problem $\left(\mathcal{P}_{2}\right)$ admits at least one solution for any $h \in L^{\infty}(\Omega)$.

For the proof of theorem 2, we observe that the main trick introduce in [7] can be adapted in our situation. Furthermore the proof needs some technical lemmas, the two next lemmas concern an a-priori estimates on the possible solutions of the following homotopic problem.

$\begin{cases}-\Delta u=\left((1-\mu) \theta+\mu \lambda_{2}\right) m_{1} u+\mu m_{2} g(u)+\mu h & \text { in } \Omega \\ \frac{\partial u}{\partial v}=0 & \text { on } \partial \Omega\end{cases}$

where $\mu \in[0,1]$ and $\theta \in] \lambda_{1}, \lambda_{2}[$ to be variable and $\lambda_{1}=\lambda_{1}\left(m_{1}\right)$.

Lemma 2 Suppose that $(\boldsymbol{A})$ and $(12)$ hold and assume that for some $\theta \in] \lambda_{1}, \lambda_{2}\left[\right.$ there exists $\mu_{n, \theta} \in[0,1]$ and $u_{n, \theta}$ be a solution of (14), for all $n$. Then we have

1) $\left(u_{n, \theta}\right)_{n}$ is a sequence of $L^{\infty}(\Omega)$ and if $\left\|u_{n, \theta}\right\|_{\infty} \rightarrow$ $+\infty$ when $n \rightarrow+\infty$, then

$$
v_{n, \theta}=\frac{u_{n, \theta}}{\left\|u_{n, \theta}\right\|_{\infty}} \rightarrow v_{\theta} \text { stongly in } C^{1}(\Omega),
$$

for some subsequence.

2) Assume that the following hypothesis holds

$$
\text { (H) } \exists \theta \in] \lambda_{1}, \lambda_{2}\left[/ \limsup _{n \rightarrow \infty} \mu_{n, \theta}=1\right.
$$

then one of the following assertions i) or ii) holds, where

i) $v_{\theta}=\psi, \psi$ is a normed $\left(\|\psi\|_{\infty}=1\right)$ eigenfunction associated to $\lambda_{2}\left(m_{1}\right)$ and

$$
\int_{\Omega} \frac{\left|g\left(u_{n, \theta}\right)\right|}{\|\left(u_{n, \theta} \|\right.} d x \rightarrow 0 \text { when } n \rightarrow+\infty .
$$

Furthermore, there exists $\eta_{1}>0, \eta_{2}>0$ such that

$$
\eta_{1}<\frac{\max \left(u_{n, \theta}\right)}{-\min \left(u_{n, \theta}\right)}<\eta_{2} \text { for n large enough. }
$$

ii) $v_{\theta}= \pm \varphi$, $\varphi$ is a normed $\left(\|\varphi\|_{\infty}=1\right)$ eigenfunction associated to $\lambda_{1}\left(\lambda_{2} m_{1}-c m_{2}\right)=1$ and

$$
\int_{\Omega} \frac{\left|g\left(u_{n, \theta}\right)+c u_{n, \theta}\right|}{\|\left(u_{n, \theta} \|\right.} d x \rightarrow 0 \text { when } n \rightarrow+\infty .
$$

Furthermore, $u_{n, \theta}$ not changes sign for $n$ large enough.
3) If $(\boldsymbol{H})$ is false, then there exists a sequence $\left(\theta_{k}\right)_{k} \subset$ ]$\lambda_{1}, \lambda_{2}\left[\right.$ and a strictly increasing sequence $\left(n_{k}\right)_{k} \subset \mathbb{N}$ such that

a) $\lim _{k \rightarrow+\infty} \theta_{k}=\lambda_{2}, \lim _{k \rightarrow \infty} \mu_{n_{k}, \theta_{k}}=1$ and $\lim _{k \rightarrow \infty}\left\|w_{k}\right\|=+\infty$ where $w_{k}=u_{n_{k}, \theta_{k}}$.

b) $\frac{w_{k}}{\left\|w_{k}\right\|} \longrightarrow \pm \varphi$ strongly in $C^{1}(\bar{\Omega})$ and

$$
\int_{\Omega} \frac{\left|g\left(w_{k}\right)+c w_{k}\right|}{\left\|w_{k}\right\|} d x \rightarrow 0 \text { when } n \rightarrow+\infty .
$$

Proof: 1) From the Anane's $L^{\infty}$-estimation [8] and the Tolksdorf's-regularity [9] we can see that $\left(u_{n, \theta}\right)_{n} \subset$ $\mathcal{C}^{1, \alpha}(\bar{\Omega})$, since the embedding $\mathcal{C}^{1, \alpha}(\bar{\Omega}) \hookrightarrow L^{\infty}(\Omega)$ is continuous for some $\alpha \in$ ] 0,1 [ independent on $n$, furthermore $v_{n, \theta}=\frac{u_{n, \theta}}{\left\|u_{n, \theta}\right\|_{\infty}}$ remains a bounded sequence in $\mathcal{C}^{1, \alpha}(\bar{\Omega})$.

By using the following compact embedding $\mathcal{C}^{1, \alpha}(\bar{\Omega}) \hookrightarrow \hookrightarrow \mathcal{C}^{1}(\bar{\Omega})$, then there exists a subsequence still denoted $\left(v_{n, \theta}\right)_{n}$ such that

$$
v_{n, \theta} \rightarrow v_{\theta} \text { stongly in } \mathcal{C}^{1}(\bar{\Omega}) \text { and }\left\|v_{\theta}\right\|_{\infty}=1 .
$$

2) According to the function $g$ satisfy the hypothesis (12), we deduce that for all $s \in \mathbb{R}$, we can write

$$
g(s)=q(s) s+r(s)
$$

where $-c \leq q(s) \leq 0$ and $\frac{r(s)}{s} \longrightarrow 0$ uniformly, when $|s| \rightarrow+\infty$. Since $u_{n, \theta}$ is a solution of $\left(\mathcal{P}_{2, \theta, \mu_{n}}\right)$, we get

$$
\begin{aligned}
\int_{\Omega} \nabla u_{n, \theta} \nabla w d x= & \int_{\Omega}\left[\left(1-\mu_{n}\right) \theta+\mu_{n} \lambda_{2}\right) m_{1} u_{n, \theta} \\
& \left.+\mu_{n} m_{2} g\left(u_{n, \theta}\right)+\mu_{n} h\right] w d x
\end{aligned}
$$

for all $w \in H^{1}(\Omega)$.

On the other hand, since $\left(u_{n, \theta}\right)_{n} \subset L^{\infty}(\Omega)$ and $q$ is a continuous function, it follows that $q\left(u_{n}\right)$ is bonded in $L^{\infty}(\Omega)$, then for a subsequence we get

$$
q\left(u_{n, \theta}\right) \rightarrow q_{\theta} \text { in } L^{\infty}(\Omega) \text { weak -*, }
$$

and

$$
\frac{\left|r\left(u_{n, \theta}\right)\right|}{\left\|u_{n, \theta}\right\|_{\infty}} \rightarrow 0 \text { strongly in } L^{\infty}(\Omega)
$$

where $-c \leq q_{\theta}(x) \leq 0$ a.e. in $\Omega$.

Dividing by $\left\|u_{n, \theta}\right\|_{\infty}$ and passing to the limit as $n \rightarrow \infty$ in 20, we get

$$
\int_{\Omega} \nabla v_{\theta} \nabla w d x=\int_{\Omega}\left(\lambda_{2} m_{1}+q_{\theta} m_{2}\right) v_{\theta} w d x \forall w \in H^{1}(\Omega) .
$$

Since $v_{\theta} \neq 0$, then 1 is an eigenvalue of Laplacain with weight $m_{q_{\theta}}=\lambda_{2} m_{1}+q_{\theta} m_{2}$.

By using the monotony property of $\lambda_{2}$ with respect to the weight, we obtain

$$
\lambda_{2}\left(m_{q_{\theta}}\right) \geq \lambda_{2}\left(\lambda_{2} m_{1}\right)=1
$$

hence $\lambda_{2}\left(m_{q_{\theta}}\right)=1$ or $\lambda_{2}\left(m_{q_{\theta}}\right)>1$.

First case: $\lambda_{2}\left(m_{q_{\theta}}\right)=1$.

So, we have

$$
q_{\theta}=0 \quad \text { and } \quad v_{\theta}=\psi
$$


Moreover, let us denotes by $F_{\lambda_{2}}$ be the eigenspace associated to $\lambda_{2}=\lambda_{2}\left(m_{1}\right)$, since $F_{\lambda_{2}}$ is a vector space of finite dimension then, we can take

$$
\eta_{1}<\min _{v \in F_{\lambda_{2}} \cap S} \frac{\max (v)}{-\min (v)} \text { and } \eta_{2}>\max _{v \in F_{\lambda_{2}} \cap S} \frac{\max (v)}{-\min (v)}
$$

where $S=\left\{v \in F_{\lambda_{2}} /\|v\|_{\infty}=1\right\}$.

It is clear to see that $\frac{\max \left(v_{n, \theta}\right)}{-\min \left(v_{n, \theta}\right)} \rightarrow \frac{\max (\psi)}{-\min (\psi)}$ when $n \rightarrow+\infty$.

It follows that for $n$ large enough

$$
\eta_{1}<\frac{\max \left(v_{n, \theta}\right)}{-\min \left(v_{n, \theta}\right)}<\eta_{2}
$$

According to 20, we get

$$
\begin{aligned}
\int_{\Omega} \frac{\left|m_{2}(x) g\left(u_{n, \theta}\right)\right|}{\left\|u_{n, \theta}\right\|_{\infty}} d x & \leq\left\|m_{2}\right\|_{\infty} \int_{\Omega}-q\left(u_{n, \theta}\right)\left|v_{n, \theta}\right| \\
& +\left\|m_{2}\right\|_{\infty} \int_{\Omega} \frac{\left|r\left(u_{n \theta}\right)\right|}{\left\|u_{n \theta}\right\|_{\infty}} d x
\end{aligned}
$$

by passage to the limit in the above inequality, we find 16 .

Second case: $\lambda_{2}\left(m_{q_{\theta}}\right)>1$.

So, we get $\lambda_{1}\left(m_{q_{\theta}}\right)=1$ and by using the strict monotony property of $\lambda_{1}$, we can see that

$$
q_{\theta}=-c \text { and } v_{\theta}=\varphi .
$$

The rest of the proof follows directly as in first case. 3) We take $\left.\left(\theta_{k}\right)_{k} \subset\right] \lambda_{1}, \lambda_{2}$ [ such that $\lim _{k \rightarrow+\infty} \theta_{k}=\lambda_{2}$. Let us denotes by $\bar{\mu}_{k}=\lim _{n \rightarrow+\infty} \mu_{k, \theta_{k}}$, as in 2) it is clear to see that

$$
\begin{cases}-\Delta u=\left[\left(\left(1-\bar{\mu}_{k}\right) \theta_{k}+\bar{\mu}_{k} \lambda_{2}\right) m_{1}+\bar{\mu}_{k} q_{\theta_{k}} m_{2}\right] v_{\theta_{k}} & \text { in } \Omega \\ \frac{\partial v_{\theta_{k}}}{\partial v}=0 & \text { on } \partial \Omega,\end{cases}
$$

we recall that $0 \leq \bar{\mu}_{k}<1,-c \leq q_{\theta_{k}} \leq 0$ and $q\left(u_{n, \theta_{k}}\right) \longrightarrow^{*}$ $q_{\theta_{k}}$ in $L^{\infty}(\Omega)$ when $n \rightarrow+\infty$, where $g(s)=q(s) s+r(s)$, $-c \leq q(s) \leq 0$ and $\frac{r(s)}{r} \rightarrow 0$ when $|s| \rightarrow+\infty$.

Let us consider the following weight

$$
\bar{M}_{k}(x)=\left(\left(1-\bar{\mu}_{k}\right) \theta_{k}+\bar{\mu}_{k} \lambda_{2}\right) m_{1}(x)+\bar{\mu}_{k} q_{\theta_{k}} m_{2}(x)
$$

we have

$$
\bar{M}_{k}(x) \leq\left(\left(1-\bar{\mu}_{k}\right) \theta_{k}+\bar{\mu}_{k} \lambda_{2}\right) m_{1}(x)
$$

because $q_{\theta_{k}} \leq 0$.

Then

$$
\begin{aligned}
\lambda_{2}\left(\bar{M}_{k}(x)\right) & \geq \lambda_{2}\left(\left(\left(1-\bar{\mu}_{k}\right) \theta_{k}+\bar{\mu}_{k} \lambda_{2}\right) m_{1}(x)\right) \\
& \geq \frac{\lambda_{2}}{\left(1-\bar{\mu}_{k}\right) \theta_{k}+\bar{\mu}_{k} \lambda_{2}}>1
\end{aligned}
$$

Thus it is clear to see that

$$
\lambda_{2}\left(\bar{M}_{k}(x)\right)=1 \text {. }
$$

Let $\bar{\mu}_{k} \rightarrow \bar{\mu}$ and $q\left(u_{n, \theta_{k}}\right) \longrightarrow^{*} \bar{q}_{0}$ in $L^{\infty}(\Omega)$ when $k \rightarrow$ $+\infty$, where $-c \leq \bar{q}_{0} \leq 0$. Then by letting $k$ tends to infinity in (23), it follows that

$$
\lambda_{1}\left(\lambda_{2} m_{1}+\bar{\mu} \bar{q}_{0} m_{2}\right)=1 .
$$

In view of lemma 1 , we get

$$
\begin{array}{ll}
\bar{\mu}=1, \bar{q}_{0}=-c & \text { and } u_{\theta_{k}} \rightarrow \pm \varphi \text { strongly in } C^{1}(\Omega) \\
& \text { when } k \rightarrow+\infty .
\end{array}
$$

On the other hand, using 20, we have

$$
\begin{aligned}
\int_{\Omega} \frac{\left|g\left(w_{k}\right)+c w_{k}\right|}{\left\|w_{k}\right\|_{\infty}} d x & =\int_{\Omega} \frac{\left|q\left(w_{k}\right) w_{k}+c w_{k}+r\left(w_{k}\right)\right|}{\left\|w_{k}\right\|_{\infty}} d x \\
& \leq \int_{\Omega}\left|q\left(w_{k}\right)+c\right| \frac{w_{k} \mid}{\left\|w_{k}\right\|_{\infty}} d x \\
& +\int_{\Omega} \frac{\left|r\left(w_{k}\right)\right|}{\left\|w_{k}\right\|_{\infty}} .
\end{aligned}
$$

Since $\frac{r(s)}{s} \rightarrow 0$ when $|s| \rightarrow \infty$, then for all $\varepsilon>0$ there exists $n_{\varepsilon, k}$ such that for all $n \geq n_{\varepsilon, k}$

$$
\int_{\Omega} \frac{\left|g\left(w_{k}\right)+c w_{k}\right|}{\left\|w_{k}\right\|_{\infty}} d x \leq \int_{\Omega}\left|\bar{q}_{\theta_{k}}+c \| v_{\theta_{k}}\right| d x+\varepsilon .
$$

If we take $\varepsilon=\frac{1}{k}, n=n_{k}=n_{\varepsilon, k}$ and we replace in the last formula, then we can see that the second member of this last inequality goes to 0 when $m$ goes to $+\infty$. Finally, we conclude by the fact that $n_{k}$ will be adjusted such that $\left\|u_{n_{k}, \theta_{k}}\right\|_{\infty}>k$ and $\left\|v_{n_{k}, \theta_{k}}-v_{\theta_{k}}\right\|_{C^{1}(\bar{\Omega})} \leq \frac{1}{k}$.

Lemma 3 Let us consider the assumptions and notations of lemma 2 We take $a \in \Omega$ and $\eta>0$ such that $B(a, \eta) \subset \Omega$.

1) Assume that the hypothesis $(\boldsymbol{H})$ holds, so, if $\left\|u_{n, \theta}\right\|_{\infty} \rightarrow+\infty$ when $n \rightarrow+\infty$ then $\limsup _{n \rightarrow+\infty} \mu_{n, \theta}=1$ and i) If $v_{\theta}=\psi$ then

$$
\lim _{n \rightarrow \infty} \int_{\substack{\text { a.e. } \\ x \in \partial B(a, \eta)}}^{1} \frac{\left|g\left(u_{n, \theta}\left(\sigma_{x}(t)\right)\right)\left\|\nabla u_{n, \theta}\left(\sigma_{x}(t)\right)\right\| x-a\right|}{\left\|u_{n, \theta}\right\|_{\infty}} d t=0
$$

where $\sigma_{x}(t)=a+t(x-a)$.

ii) If $v_{\theta}= \pm \varphi$ then

$$
\lim _{n \rightarrow \infty} \int_{0}^{1} \frac{\mid g\left(u_{n, \theta}\left(\sigma_{x}(t)\right)\right)+c u_{n, \theta}\left(\sigma_{x}(t)\left\|\nabla u_{n, \theta}\left(\sigma_{x}(t)\right)\right\| x-a \mid\right.}{\left\|u_{n, \theta}\right\|_{\infty}} d t=0
$$

2) Assume that $(\boldsymbol{H})$ is false then we have

$$
\lim _{n \rightarrow \infty} \int_{\substack{\text { a.e. } \\ \text { a }}} \frac{\mid g\left(w_{k}\left(\sigma_{x}(t)\right)\right)+c w_{k}\left(\sigma_{x}(t)\left\|\nabla w_{k}\left(\sigma_{x}(t)\right)\right\| x-a \mid\right.}{\left\|w_{k}\right\|_{\infty}} d t=0
$$

Proof: We only show the relation 25 since the proof of the 26 and 27) one proceeds in the same way. Firstly we have

$$
\int_{B(a, \eta)} \frac{\left|g\left(u_{n, \theta}\right)\right|}{\left\|u_{n, \theta}\right\|_{\infty}} d x \leq \int_{\Omega} \frac{\left|g\left(u_{n, \theta}\right)\right|}{\left\|u_{n, \theta}\right\|_{\infty}} d x .
$$

According to 16, we obtain

$$
\lim _{n \rightarrow \infty} \int_{B(a, \eta)} \frac{\left|g\left(u_{n, \theta}\right)\right|}{\left\|u_{n, \theta}\right\|_{\infty}} d x=0
$$


Which gives by passing in spherical coordinates

$$
\begin{aligned}
\lim _{n \rightarrow \infty} \int_{[0, \pi]^{n}} & \int_{0}^{2 \pi} \int_{0}^{\eta} t^{N-1} \frac{\left|g\left(u_{n, \theta}(a+t \omega)\right)\right|}{\left\|u_{n, \theta}\right\|_{\infty}} \\
& \prod_{j=1}^{N-2}\left(\sin \theta_{j}\right)^{N-1-j} d \theta_{j} d \theta_{N-1} d t=0
\end{aligned}
$$

where, $\omega=\frac{x-a}{\eta} \in \partial B(0,1)$.

The above equality imply

$$
\begin{array}{ll}
\frac{\left|g\left(u_{n, \theta}\left(\sigma_{x}(\tau)\right)\right)\right|}{\left\|u_{n, \theta}\right\|_{\infty}} \rightarrow 0 \quad \text { when } n \rightarrow \infty & \text { a.e. } x \in \partial B(a, \eta) \text { and a.e. } \tau \in[0,1
\end{array}
$$

By using 12 we can see that $g$ satisfy the following growth condition:

$$
|g(s)| \leq a|s|+b \text {, for some positive reals } a, b \text { and } \forall s \in \mathbb{R}
$$

hence $\left(\frac{\left|g\left(u_{n, \theta}\left(\sigma_{x}(.)\right)\right)\right|}{\left\|u_{n, \theta}\right\|_{\infty}}\right)_{n}$ and $\left(\frac{\left|\nabla\left(u_{n, \theta}\left(\sigma_{x}(.)\right)\right)\right|}{\left\|u_{n, \theta}\right\|_{\infty}}\right)_{n}$ are bounded in $L^{\infty}([0,1])$.

By using the Lebesgue dominated convergence theorem, we conclude this proof.

Lemma 4 1) If $G$ satisfy $-c<\limsup _{s \rightarrow+\infty} \frac{2 G(s)}{s^{2}} \leq 0$. then for all $r \in] 0,1\left[\right.$, there exists $\rho_{r}>-c\left(1-r^{2}\right)$ and a sequence of positive real numbers $\left(S_{n}\right)$ such that

$$
\lim _{n \rightarrow+\infty} S_{n}=+\infty \text { and } \lim _{n \rightarrow+\infty} \frac{2 G\left(S_{n}\right)-2 G\left(r S_{n}\right)}{S_{n}^{2}}=\rho_{r} .
$$

2) If $G$ satisfy $-c<\limsup _{s \rightarrow+\infty} \frac{2 G(s)}{s^{2}} \leq 0$ and

$-c<\liminf _{s \rightarrow+\infty} \frac{2 G(s)}{s^{2}}<0$, then for all $\left.r \in\right] 0,1[$, there exists $\rho_{r}>-c\left(1-r^{2}\right)$ and a sequence of positive real numbers $\left(S_{n}^{\prime}\right)$ such that

$$
\begin{aligned}
\lim _{n \rightarrow+\infty} S_{n}^{\prime}=+\infty, & \lim _{n \rightarrow+\infty} \frac{2 G\left(S_{n}^{\prime}\right)}{S_{n}^{\prime 2}}=\rho \\
& \text { and } \lim _{n \rightarrow+\infty} \frac{2 G\left(S_{n}^{\prime}\right)-2 G\left(r S_{n}^{\prime}\right)}{S_{n}^{\prime 2}}=\rho_{r} .
\end{aligned}
$$

The same conclusion is obtained when we replace $+\infty$ par $-\infty$, in this case we should note $\rho^{\prime}$ and $\rho_{r}$ in place of $\rho$ and $\rho_{r}$ respectively.

Proof: We pose $L_{1}=\liminf _{s \rightarrow+\infty} \frac{2 G(s)}{s^{2}}$ and $L_{2}=$ $\limsup _{s \rightarrow+\infty} \frac{2 G(s)}{s^{2}}$.

We distinguish the following two cases:

Case1: If $L_{1}=L_{2}$, it is enough to take $\rho=L_{2}$ and $\rho_{r}=\left(1-r^{2}\right) L_{2}$.

Case2: If $L_{1}<L_{2}$, then we choose $\left.\rho \in\right] L_{1}, L_{2}$ [ neighbor of $L_{2}$ in such a way that:

$$
\rho-r^{2} L_{2}>-c\left(1-r^{2}\right)
$$

According to the definition of $L_{1}$ and $L_{2}$, we conclude that, there existence a sequence $\left(S_{n}\right)$ such that $\lim _{n \rightarrow+\infty} S_{n}=+\infty$ and $\frac{2 G\left(S_{n}\right)-2 G\left(r S_{n}\right)}{S_{n}^{2}}=\rho$. We put

$$
\liminf _{n \rightarrow+\infty} \frac{2 G\left(S_{n}\right)-2 G\left(r S_{n}\right)}{S_{n}^{2}}=\rho-r^{2} \limsup _{n \rightarrow+\infty} \frac{2 G\left(r S_{n}\right)}{r S_{n}^{2}} .
$$

Since $\limsup _{n \rightarrow+\infty} \frac{2 G\left(r S_{n}\right)}{\left(r S_{n}\right)^{2}} \leq L_{2}$, then we have:

$$
\rho_{r} \geq \rho-r^{2} L_{2}>-c\left(1-r^{2}\right) .
$$

\section{Proof of Theorem 2}

Our purpose now, consists in building in $C(\bar{\Omega})$ an open bounded set $\mathcal{O}$ such that, there exist $\theta \in] \lambda_{1}, \lambda_{2}[$ such that, no solution of 14 with $\mu \in[0,1$ [ occurs on the boundary $\partial \mathcal{O}$. Homotopy invariance of the degree then yields the conclusion. The set $\mathcal{O}$ will have the following form

$$
\mathcal{O}=\mathcal{O}_{S, T}=\{u \in \mathcal{C}(\bar{\Omega}) ; \quad T<u<S\},
$$

where, $S$ and $T$ satisfy $T<0<S$.

Firstly, according to, 13 we will assume the following hypothesis holds

$$
-c<\limsup _{|s| \rightarrow \infty} \frac{2 G(s)}{s^{2}} \text { and } \liminf _{s \rightarrow+\infty} \frac{2 G(s)}{s^{2}}<0
$$

An analogous proof will be adapted to the hypothesis $\left(13_{-}\right)$where $(13)$ represents $\left(13_{+}\right)$or $\left(13_{-}\right)$. Assume by contradiction, that for all $\theta \in] \lambda_{1}, \lambda_{2}$ [ and for all $S, T(T<0<S)$, there exists $\mu=\mu_{\theta, S, T} \in[0,1[$ and $u=u_{\theta, S, T} \in \partial \mathcal{O}$ such that $u$ is a solution of 14 which gives

$$
\max (u)=S \text { or } \min (u)=T .
$$

According to $\left(13_{+}\right)$, then owing to lemma 4 , there exists two sequence $\left(T_{n}\right)$ and $\left(S_{n}\right)$ such that

$$
\lim _{n \rightarrow+\infty} T_{n}=-\infty, \lim _{n \rightarrow+\infty} \frac{2 G\left(T_{n}\right)-2 G\left(r T_{n}\right)}{T_{n}^{2}}=\rho_{r}^{\prime}
$$

$\lim _{n \rightarrow+\infty} S_{n}=+\infty, \lim _{n \rightarrow+\infty} \frac{2 G\left(S_{n}\right)}{S_{n}^{2}}, \lim _{n \rightarrow+\infty} \frac{2 G\left(S_{n}\right)-2 G\left(r S_{n}\right)}{S_{n}^{2}}=\rho_{r}$

where $r=\frac{\min \varphi}{\max \varphi}$ and $\rho, \rho_{r}$ and $\rho_{r}^{\prime}$ provides from lemma 4

So, we remark that, without loss of generality we can assume that

$$
\frac{S_{n}}{-T_{n}} \leq \eta_{1}
$$

We use the following notations:

$$
T=T_{n}, S=S_{n}, \mu_{n, \theta}=\mu_{\theta, T, S} \text { and } u_{n, \theta}=u_{\theta, T, S} .
$$

According to 29 and 31, we obtain

$$
\left\|u_{n, \theta}\right\|_{\infty} \rightarrow+\infty \text { when } n \rightarrow+\infty .
$$

We will distinguish two cases.

First case: We assume that the hypothesis $(\mathbf{H})$ is satisfied (c.f. lemma 2).

According to lemma 2 we get

$$
v_{n, \theta}=\frac{u_{n, \theta}}{\left\|u_{n, \theta}\right\|_{\infty}} \rightarrow v_{\theta} \text { where } v_{\theta}=\psi \text { or } v_{\theta}= \pm \varphi \text {. }
$$


i) If $v_{\theta}=\psi$, we assert that for every $\mathrm{n}$ large enough we have

$$
\max \left(u_{n, \theta}\right)=S_{n}
$$

Indeed, if not we have

$$
\max \left(u_{n, \theta}\right)<S_{n} \text { and } \min \left(u_{n, \theta}\right)=T_{n}
$$

thus,

$$
\frac{\max \left(u_{n, \theta}\right)}{-\min \left(u_{n, \theta}\right)}<\frac{S_{n}}{-S_{n}} \leq \eta_{1}
$$

which gives a contradiction from the definition of $\eta_{1}$ given in lemma 2. On the other hand, we have $u_{n, \theta}$ changes sign on $\Omega$, so there exists $x_{n} \in \bar{\Omega}, y_{n} \in \Omega$ such that

$$
u_{n, \theta}\left(x_{n}\right)=S_{n} \text { and } u_{n, \theta}\left(y_{n}\right)=0,
$$

we write

$$
\begin{aligned}
\frac{2 G\left(S_{n}\right)}{S_{n}^{2}}= & \frac{2 G\left(u_{n, \theta}\left(x_{n}\right)\right)-2 G\left(u_{n, \theta}\left(x_{n}\right)\right)}{\left\|u_{n, \theta}\right\|_{\infty}^{2} \max \left(v_{n, \theta}\right)^{2}} \\
& =\frac{2}{\max \left(v_{n, \theta}\right)^{2}} \int_{\mathcal{C}_{n}} \frac{d\left(G \circ u_{n, \theta}\right)}{\left\|u_{n, \theta}\right\|_{\infty}^{2}}
\end{aligned}
$$

where

$$
d\left(G \circ u_{n, \theta}\right)\left(\mathcal{C}_{n}\right)=g\left(u_{n, \theta}\left(\mathcal{C}_{n}\right)\right) \nabla u_{n, \theta}\left(\mathcal{C}_{n}\right) \cdot \mathcal{C}_{n}^{\prime} \text {, a.e. }
$$

and $\mathcal{C}_{n}$ is a $\mathcal{C}^{1}$ with morsels line which connects extremity $x_{n}$ and $y_{n}$.

According to lemma 3, we have

$$
\lim _{n \rightarrow+\infty} \int_{\mathcal{C}_{n}} \frac{d\left(G \circ u_{n, \theta}\right)}{\left\|u_{n, \theta}\right\|_{\infty}^{2}}
$$

Since $\max \left(v_{n, \theta}\right) \rightarrow \max (\psi)$ when $n \rightarrow+\infty$, we deduce that

$$
\rho=\lim _{n \rightarrow+\infty} \frac{2 G\left(S_{n}\right)}{S_{n}^{2}}=0,
$$

which is a contradiction since $\rho \in]-c, 0[$.

ii) If $v_{\theta}= \pm \varphi$ : then, for $n$ large enough $u_{n, \theta}$ not changes sign on $\Omega$.

so,

$$
\max \left(u_{n, \theta}\right)=S_{n} \text { or } \min \left(u_{n, \theta}\right)=T_{n} .
$$

Assume that $\max \left(u_{n, \theta}\right)=S_{n}$ (the same gait will be used for the case $\left.\min \left(u_{n, \theta}\right)=T_{n}\right)$, so it is clear to see that $v_{n, \theta}=+\varphi$ and $\min \left(u_{n, \theta}\right)>0$ for $n$ large enough. We put

$$
\bar{g}(s)=g(s)+c s, \bar{G}(s)=\int_{0}^{s} \bar{g}(s) d s .
$$

Let $x_{n}, y_{n} \in \bar{\Omega}$ such that $u_{n, \theta}\left(x_{n}\right)=S_{n}$ and $u_{n, \theta}\left(y_{n}\right)=$ $\min \left(u_{n, \theta}\right)$.

We write

$$
\begin{aligned}
\frac{\bar{G}\left(S_{n}\right)-\bar{G}\left(r_{n} S_{n}\right)}{S_{n}^{2}}= & \frac{2 \bar{G}\left(u_{n, \theta}\left(x_{n}\right)\right)-2 \bar{G}\left(u_{n, \theta}\left(x_{n}\right)\right)}{\left\|u_{n, \theta}\right\|_{\infty}^{2}} \\
& =\int_{\mathcal{C}_{n}} \frac{d\left(\bar{G} \circ u_{n, \theta}\right)}{\left\|u_{n, \theta}\right\|_{\infty}^{2}}
\end{aligned}
$$

where $r_{n}=\frac{\min \left(u_{n, \theta}\right)}{\max \left(u_{n, \theta}\right)} \rightarrow r=\frac{\min (\varphi)}{\max (\varphi)}$.

Using the Lemma 3, we deduce that

$$
\lim _{n \rightarrow+\infty} \int_{\mathcal{C}_{n}} \frac{d\left(\bar{G} \circ u_{n, \theta}\right)}{\left\|u_{n, \theta}\right\|_{\infty}^{2}}=0
$$

On the other hand, it is easy to verify that

$$
0=\lim _{n \rightarrow+\infty} \frac{\bar{G}\left(S_{n}\right)-\bar{G}\left(r_{n} S_{n}\right)}{S_{n}^{2}}=\frac{\rho_{r}+c\left(1-r^{2}\right)}{2}>0
$$

which gives a contradiction.

Second case: Assume that the hypothesis $(\mathbf{H})$ is not verified, so for all $\theta \in] \lambda_{1}, \lambda_{2}$ [ such that

$\limsup \mu_{n, \theta}<1$.

We take a sequence $\left(\theta_{k}\right)$ such that

$$
\lim _{k \rightarrow+\infty} \theta_{k}=\lambda_{2}
$$

and we consider the subsequences

$$
\left(T_{n_{k}}\right)_{k},\left(S_{n_{k}}\right)_{k} \text { and } w_{k}=u_{n_{k}, \theta_{k}} \text {. }
$$

Similarly, as in the second point of the previous case we obtain a contradiction. This completes the proof of theorem 2 .

\section{Nonresonance between the first two Eigencurves}

In this section we will prove an existence result for problem $(\mathcal{P})$. We need more restrictive hypotheses on the nonlinearities $g$ and $G$.

$$
\begin{aligned}
& \left(\mathbf{A}_{g}\right) \quad \beta_{1} \leq \liminf _{s \rightarrow \pm \infty} \frac{g(s)}{s} \leq \limsup _{s \rightarrow \pm \infty} \frac{g(s)}{s} \leq \beta_{2} \\
& \left(\mathbf{A}_{G}^{ \pm}\right) \quad \beta_{1}<\limsup _{|s| \rightarrow \infty} \frac{2 G(s)}{s^{2}} ; \liminf _{s \rightarrow+\infty \text { or }-\infty} \frac{2 G(s)}{s^{2}}<\beta_{2}
\end{aligned}
$$

where $\left(\beta_{1}, \beta_{2}\right) \in \mathbb{R}^{2}$ with $\beta_{2}-\beta_{1}=c$ and $c$ is given in 10 .

Theorem 3 Let $m_{1}, m_{2} \in M^{+}(\Omega)$. Assume that $(A),\left(A_{g}\right)$ and $\left(A_{G}^{ \pm}\right)$holds. Moreover, if $\left(\alpha, \beta_{1}\right) \in \mathcal{C}_{1}$ and $\left(\alpha, \beta_{2}\right) \in \mathcal{C}_{2}$, where $\alpha \geq \lambda_{2}\left(m_{1}\right)$ or $\alpha \leq \lambda_{-} 2\left(m_{1}\right)$. Then the problem $(\mathcal{P})$ has at least one nontrivial weak solution $u \in H^{1}(\Omega)$ for any given $h \in L^{\infty}(\Omega)$.

Proof: The problem $(\mathcal{P})$ can be written in the following equivalent form

$$
\left(\mathcal{P}_{e}\right)\left\{\begin{aligned}
-\Delta u & =\widetilde{m}|u|^{p-2} u+m_{2} \widetilde{g}(u)+h & & \text { in } \Omega \\
\frac{\partial u}{\partial v} & =0 & & \text { on } \partial \Omega,
\end{aligned}\right.
$$

where

and

$$
\widetilde{g}(s)=g(s)-\beta_{2} s,
$$

$$
\widetilde{m}=\alpha m_{1}+\beta_{2} m_{2}
$$

Since $\left(\alpha, \beta_{2}\right) \in \mathcal{C}_{2}$, then 1 is the second eigenvalue of laplacian operator with weight $\widetilde{m}$ relating to Neumann boundary conditions. In view of theorem 2 , there exists at least one weak solution $u \in H^{1}(\Omega)$ of 
the problem $\left(\mathcal{P}_{e}\right)$ for all $h \in L^{\infty}(\Omega)$ if the function $\widetilde{g}$ and his potential $\widetilde{G}$ satisfy the two conditions 12 and 13. Indeed, in view of $\left(\mathbf{A}_{g}\right)$

$$
\beta_{1}-\beta_{2} \leq \lim \inf _{|s| \rightarrow \infty}\left(\frac{g(s)}{s}-\beta_{2}\right) \leq 0
$$

thus

$$
-c \leq \lim \inf _{|s| \rightarrow \infty}\left(\frac{\widetilde{g}(s)}{s}\right) \leq 0 .
$$

Consequently, $\widetilde{g}$ satisfy 12 .

On the other hand, using $\left(\mathbf{A}_{G}^{ \pm}\right)$, we have

$$
\beta_{1}-\beta_{2}<\limsup _{|s| \rightarrow \infty}\left(\frac{2 G(s)}{s^{2}}-\beta_{2}\right) ; \liminf _{s \rightarrow+\infty}\left(\frac{2 G(s)}{s^{2}}-\beta_{2}\right)<0
$$

hence

$$
-c<\limsup _{|s| \rightarrow \infty}\left(\frac{2 \widetilde{G}(s)}{s^{2}}\right) ; \liminf _{s \rightarrow+\infty}\left(\frac{2 \widetilde{G}(s)}{s^{2}}\right)<0
$$

which means that $\widetilde{G}$ satisfy 13 .

Finally, since the problem $\left(\mathcal{P}_{e}\right)$ is a equivalent to the problem $(\mathcal{P})$. Then the problem $(\mathcal{P})$ has at least one nontrivial weak solution $u \in H^{1}(\Omega)$ for any given $h \in L^{\infty}(\Omega)$.

\section{References}

1. D. Del Santo et P. Omari, Nonresonance conditions on the potential for a semilinear elliptic problem. J. Diff. Equ. pp.
120-138, (1994).

2. J.-P. Gossez and P. Omari, A necessary and sufficient condition of nonresonance for a semilinear Neumann problem, Proc. Amer. Math. Soc. Vol. 114, No. 2 (Feb., 1992), pp. 433-442.

3. A. Dakkak, A. Anane, Nonresonance Conditions on the Potential for a Neumann Problem, proceedings of the international conference held in Fez, partial differential equations pp. 85-102.

4. A. Dakkak, Etude sur le spectre et la résonance pour des problèmes elliptiques de Neumann, Thèse de 3eme cycle, Faculté des sciences, Oujda, Maroc, (1995).

5. A. Dakkak, M. Hadda, Eigencurves of the p-Laplacian with weights and their asymptotic behavior EJDE, Vol 2007(2007), No. 35, pp. 1-7.

6. A. Dakkak and M. Moussaoui On the second eigencurve for the p-laplacian operator with weight, Bol. Soc. Paran. Mat., (3s.) v. 351 (2017): 281289.

7. J.-P. Gossez and P. Omari, On a semilinear elliptic Neumann problem with asymmetric nonlinearities. Transactions of Amer. Math. Soc. Volume 347, Number 7, pp. 2553-2562, July 1995.

8. A. Anane, Etude des valeurs propres et de la résonance pour l'operateur p-Laplacien, these de Doctorat, Universite Libre de Bruxelles, (1988).

9. P.Tolksdorf, Regularity for more general class of quasilinear elliptic equation J.Diff. Eq. 8, (1983), pp. 773-817. 